\title{
Incidence of apoptosis in granulosa cells from immature human follicles
}

\author{
A. L. Mikkelsen, E. Høst and S. Lindenberg \\ The Fertility Clinic, Institute for Human Reproduction, Symbion, Fruebjergvej 3, \\ DK-2100 Copenhagen, Denmark
}

The aim of this study was to investigate the incidence of apoptosis in granulosa cells from immature human follicles undergoing in vitro maturation (IVM) and to compare the incidence of apoptotic granulosa cells (i) between FSHprimed and unprimed normal ovaries and (ii) between polycystic and normal ovaries. Furthermore, the incidence of apoptosis was related to maturation and subsequent fertilization and cleavage of the oocytes from the corresponding ovary. Seventy women undergoing 70 IVM cycles were included. Group 1 consisted of patients with normal ovaries $(n=52)$ and group 2 consisted of patients with polycystic ovaries $(n=18)$. Patients in group 1 were subdivided into two groups according to priming with FSH before aspiration. In group 1a ( $n=27$ cycles) oocytes were obtained in unstimulated cycles. In group 1 b ( $n=25$ cycles) oocytes were obtained after priming with recombinant FSH for 3 days initiated on day 3 after spontaneous menstruation. In group 2 all patients were primed with recombinant $\mathrm{FSH}$ for 3 days before aspiration. Aspiration was performed transvaginally and cumulus-enclosed oocytes were matured for $28-30 \mathrm{~h}$ before fertilization. Granulosa cells were collected from follicular aspirates. An APOPTAG ${ }^{\circledR}$ detection kit was used to stain the granulosa cells and to detect apoptosis. The incidence of apoptosis in granulosa cells was decreased in follicles from $\mathrm{FSH}$-primed normal ovaries compared with follicles from unprimed normal ovaries and FSH-primed polycystic ovaries. No difference was found between granulosa cells from $\mathrm{FSH}$ primed polycystic ovaries and granulosa cells from unstimulated normal ovaries. No differences in maturation rate, fertilization rate, cleavage rate and implantation rate were observed when oocytes from a polycystic ovary were compared with oocytes from an unstimulated normal ovary. In unstimulated cycles, the ovaries were grouped according to the presence of a dominant follicle. The incidence of apoptosis was significantly higher in granulosa cells from an ovary without a dominant follicle compared with granulosa cells from an ovary with a dominant follicle. The rates of maturation, fertilization and cleavage did not differ between the two groups.

\section{Introduction}

Apoptosis is involved in regulation and selection of human ova from the primordial follicle pool (De Pol et al., 1997) and is also thought to play a major role in the depletion of oocytes from ovarian tissue during ageing (Wu et al., 2000). Apoptosis can be initiated in at least four different cell compartments in follicular development (theca cells, granulosa cells, cumulus cells and in the oocyte itself). Apoptosis in granulosa cells and cumulus cells is associated with follicular atresia during natural (Yuan and Giudice, 1997) and stimulated (Nakahara et al., 1997a; Oosterhuis et al., 1998; Høst et al., 2000) ovarian cycles. However, the mechanism by which oocytes and granulosa cells interact to escape or promote apoptosis is poorly understood (Driancourt and Thuel, 1998).

A correlation between low incidence of apoptosis in cumulus-granulosa cells and improved embryo development is suggested when oocytes and cumulus-granulosa cells are obtained after controlled ovarian hyperstimulation (Seifer et

Email:alm@ciconia.dk al., 1996; Nakahara et al., 1997a; Oosterhuis et al., 1998). Lower incidence of apoptosis from a single cumulus-oocyte complex correlates with maturation to metaphase II and better outcome of the corresponding oocyte in terms of fertilization rate (Høst et al., 2000) and in vitro development (Nakahara et al., 1997b). These findings indicate that the incidence of apoptosis in granulosa and cumulus cells may be useful to assess the fertility potential of oocytes recruited after controlled ovarian stimulation for in vitro fertilization (IVF).

Increased efforts have been devoted to in vitro maturation (IVM) of human oocytes (Trounson et al., 1998; Cha et al., 2000; Mikkelsen et al., 2000). However, apoptosis has not been reported in a human IVM embryo transfer programme. The aim of the present study was to investigate the incidence of apoptotic changes in granulosa cells from immature follicles in women undergoing IVM. The incidence of apoptosis was compared between normal and polycystic ovaries and related to rates of maturation, fertilization and cleavage of the oocytes. In unstimulated cycles, the ovaries were grouped according to the presence of a dominant follicle. The incidence of apoptosis was compared between the two groups and the rates of maturation, fertilization and 
cleavage were compared between oocytes originating from the ovary with a dominant follicle and oocytes originating from the ovary without a dominant follicle.

\section{Materials and Methods}

\section{Subjects}

The patients were recruited in the immature oocyte retrieval programme from patients referred for IVF-ICSI (intracytoplasmic sperm injection) due to male factor infertility, tubal factor infertility or polycystic ovary syndrome (PCOS). The procedure was explained to the patients and they gave informed consent before the procedure. Seventy IVM cycles from 70 consecutive women enrolled in the IVM programme were included in the study.

Group 1 included patients with normal ovaries $(n=52)$ referred for IVM due to male factor infertility $(n=34)$ or tubal disease $(n=18)$. Group 2 included patients with polycystic ovaries $(n=18)$, defined as $>10$ follicles in one plane (Adams et al., 1985). All the women were $<38$ years of age with a body mass index between $18 \mathrm{~kg} \mathrm{~m}^{-2}$ and $29 \mathrm{~kg} \mathrm{~m}^{-2}$. The basal FSH concentration was $<15 \mathrm{iu} \mathrm{l}^{-1}$ (Scott et al., 1989) and the basal inhibin B concentration was $>45 \mathrm{pg} \mathrm{ml}^{-1}$ (Seifer et al., 1997). Patients with infertility caused by endocrine abnormalities such as hyperprolactinaemia and patients with one ovary or ovarian endometriosis were excluded. Patients with possible poor quality oocytes (patients with a low $(<20 \%)$ cleavage rate after controlled ovarian hyperstimulation and IVF or intracytoplasmic hyperstimulation) were also excluded. Moreover, patients who had previously failed more than three times to conceive with IVF-ICSI were not included. The cycle was cancelled (on day 3 of the cycle) if an ovarian cyst $>10 \mathrm{~mm}$ in diameter was observed.

The IVM-IVF and embryo transfer procedure had been approved by the local ethics committee and written informed consent was obtained from all participants.

\section{Experimental design}

Women with normal ovaries were subdivided into two groups. Group 1a included 27 women undergoing unstimulated cycles. Oocyte recovery was performed on the day after a follicle $10 \mathrm{~mm}$ in diameter and an endometrium of $5 \mathrm{~mm}$ in thickness could be detected by ultrasonography (Mikkelsen et al., 2000). Group $1 \mathrm{~b}$ included 25 women primed with 150 iu recombinant FSH day $^{-1}$ (Gonal-F; Serono, Genova) for 3 days, initiated on day 3. Oocyte recovery was performed on days 8-9 after deprivation of FSH for 2-3 days (Mikkelsen et al., 1999).

All women with polycystic ovaries were primed with 150 iu recombinant $\mathrm{FSH}$ for 3 days initiated on day 3, and oocyte recovery was performed on days 8-9 after deprivation of FSH for 2-3 days (group 2).

For each patient the incidence of apoptosis in granulosa cells was recorded for each ovary. Granulosa cells from the aspirated follicles were collected for each ovary. In unstimulated cycles with a dominant follicle the granulosa cells from this follicle were separated and not pooled with granulosa cells from the remaining follicles.

In unstimulated cycles the ovaries were grouped according to the presence of a dominant follicle. The following factors were recorded for each ovary: the number of oocytes retrieved, the number of oocytes reaching metaphase II, the number of oocytes cleaved and the number of embryos transferred.

Oocytes from unprimed normal ovaries (group 1a) and FSH-primed polycystic ovaries (group 2) were matured in the same medium and in these groups the rates of maturation, fertilization, cleavage and implantation were compared.

\section{Ultrasonography}

An ultrasound examination was performed on day 3, days 6-7 and either once a day or at 2 day intervals until the day of aspiration using a $7.5 \mathrm{MHz}$ transvaginal transducer (B \& K Medical, Gentofte). The follicular diameter was calculated as the mean of the longest follicular axis and the axis perpendicular to it. A clinical pregnancy was defined as evidence of intrauterine fetal heart activity after embryo transfer visualized by ultrasonography.

\section{Oocyte recovery, maturation, fertilization and embryo culture}

These procedures have been described in detail previously by Smith et al. (2000) and Mikkelsen et al. (2000). In brief, oocyte recovery was performed transvaginally with a $17 \mathrm{G}$ single lumen needle (K-OPSC1225; Cook, Queensland) connected to a syringe to induce the aspiration vacuum, and the follicular aspirates were transferred into tubes containing Ham's F-10 medium with heparin (Life Technologies, Roedovre) at $37^{\circ} \mathrm{C}$. The aspirates were filtered (Falcon 1060; $70 \mu \mathrm{m}$ mesh size) to remove erythrocytes and small cellular debris. The oocytes were isolated under a stereomicroscope and washed twice. The oocytes and their cumulus investments were classified as follows: multilayered cumulus, sparse cumulus, nude or atretic. Only oocytes that were classified as having a multilayered or sparse cumulus were used for the experiments.

Oocytes from groups $1 \mathrm{a}$ and 2 were matured in TCM199 (Sigma, Roedovre) supplemented with $0.3 \mathrm{mmol}$ sodium pyruvate $\mathrm{I}^{-1}$ (Sigma), $1500 \mathrm{iu}$ penicillin $\mathrm{G} \mathrm{ml}^{-1}$ (Sigma), $50 \mathrm{mg}$ streptomycin sulphate $\mathrm{ml}^{-1}$ (Sigma), $1 \mathrm{mg}$ oestradiol $\mathrm{ml}^{-1}$ (Sigma), $0.075 \mathrm{iu}$ recombinant $\mathrm{FSH} \mathrm{ml}{ }^{-1}$ (Gonal-F; Serono), 0.5 iu hCG $\mathrm{ml}^{-1}$ (Profasi; Serono) and heat-inactivated serum from the patient $(10 \%)$. Oocytes were cultured separately in $25 \mu \mathrm{l}$ droplets of culture medium under paraffin oil at $37^{\circ} \mathrm{C}$ in $5 \% \quad \mathrm{CO}_{2}$ and humidified air for 28-30 h.

After maturation the oocytes were denuded with hyaluronidase (IVF Science, Gothenburg) and mechanical 
pipetting. Motile spermatozoa were prepared by Puresperm ${ }^{\mathrm{TM}}$ (Cryos, Copenhagen) gradient separation. For ICSI, denuded oocytes were placed individually into droplets of sperm prep medium (Medi-Cult, Copenhagen) and $2 \mu \mathrm{l}$ sperm suspension was placed into a $10 \mu \mathrm{l}$ droplet of polyvinyl peroxidase (IVF Science). ICSI was performed on all metaphase II oocytes. The oocytes were then cultured in Falcon Petri dishes in droplets of IVF medium (Medi-Cult) until day 2 or 3 after fertilization at which time suitable embryos (maximum two embryos) were transferred into the uterus.

Images were recorded at $\times 300$ magnification at the start of IVM, after 22-23 $\mathrm{h}$ of IVM and at the end of IVM (28-30 $\mathrm{h}$ from the start of aspiration) after the oocytes were prepared for ICSI. All of the oocytes were inseminated by ICSI to confirm the presence of the first polar body microscopically, which was the criterion used to classify oocytes as matured to metaphase II. Fertilization was defined as the presence of two pronuclei.

Endometrial priming consisted of administration of oestradiol (2 mg orally three times per day) started on the day of oocyte retrieval. On day 2 after aspiration, treatment with intravaginal progesterone suppositories was initiated (100 mg three times a day) and continued until the pregnancy test. Oestrogen and progesterone treatment were continued if the pregnancy test was positive until day 50 of gestation.

\section{Granulosa cell preparation, analysis and assessment of apoptosis}

The granulosa cells were collected from the follicular aspirates during aspiration and washed twice in Earle's balanced salt solution (EBSS; Medi-Cult). The pellet with granulosa cells was pipetted onto a Silan-coated microscope slide and a thin smear was prepared. Slides were air-dried and fixed in 96\% (v/v) ethanol for $5 \mathrm{~min}$. An APOPTAG $^{\circledR}$ in situ apoptosis detection kit-peroxidase (INTERGEN Oxford, catalogue no S7100-KIT) with some modifications for staining of the granulosa cells for the detection of apoptosis (Høst et al., 1999) was used. The APOPTAG $^{\circledR}$ kit is designed to label the free $3^{\prime}-\mathrm{OH}$ DNA termini in situ with chemically labelled and unlabelled nucleotides. The nucleotides contained in the reaction buffer are added enzymatically to the DNA by terminal deoxynucleotidyl transferase (TdT). TdT catalyses a template-independent addition of nucleotide triphosphates to the 3'-OH ends of double- or single-stranded DNA. The incorporated nucleotides from an oligomer composed of digoxigenin are allowed to bind an anti-digoxigenin antibody that is conjugated to peroxidase. The localized peroxidase enzyme then catalytically generates an intense signal from chromogenic substrates. A control without enzyme was used to determine background staining and mixed water was used in reaction buffer instead of TdT enzyme as a negative control. Background staining was $<3 \%$.

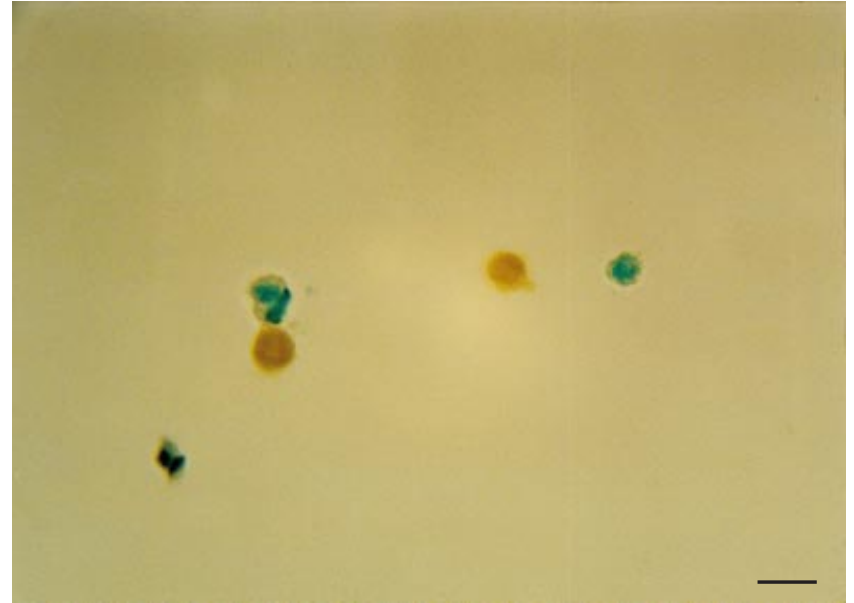

Fig. 1. Micrograph showing two granulosa cells with apoptosis (brown coloured) and two granulosa cells without apoptosis (green coloured). Scale bar represents $10 \mathrm{~nm}$.

Apoptosis and morphology were scored according to peroxidase-3,3'diaminobenzidine staining. About 300 granulosa cells on each slide were analysed to evaluate the degree of apoptosis. The cells were viewed under a microscope by oil immersion at $\times 1000$ magnification and bright light field illumination (Fig. 1).

The slides were assessed by the same observer twice, as the slides were reassessed randomly. The intra-observation variation was $<2 \%$.

The incidence of apoptosis was examined on a per ovary basis. The apoptotic index is the degree (\%) of apoptotic activated granulosa cells per ovary.

\section{Statistical analysis}

Statistical analyses were performed using chi-squared test or Fisher's exact test. Mann-Whitney rank sum test or Kruskal-Wallis test was used for comparison between apoptosis in the cumulus cells. Values were considered significant at $P<0.05$.

\section{Results}

The incidence of apoptosis in granulosa cells from FSHprimed normal ovaries was lower than the incidence of apoptosis in granulosa cells from unprimed normal ovaries and FSH-primed polycystic ovaries. No difference in the incidence of apoptosis was found between unstimulated normal ovaries and FSH-primed polycystic ovaries (Table 1). More oocytes were obtained from polycystic ovaries (median 8, range 4-17 oocytes per aspiration) compared with unstimulated normal ovaries (median 5, range 2-9 oocytes per aspiration). A total of 153 oocytes was recovered from polycystic ovaries and, of these, 88 (58\%) cumulus-enclosed oocytes were used in the experiment. 
Table 1. Incidence of apoptosis in granulosa cells from unstimulated follicles of normal human ovaries (group 1a), stimulated follicles from normal ovaries (group 1b) and stimulated follicles from polycystic ovaries (group 2)

\begin{tabular}{lccc}
\hline & Group 1a & Group 1b & Group 2 \\
\hline Number of IVM cycles & 27 & 25 & 18 \\
Apoptosis median (\%) & $45.9^{\mathrm{a}}$ & $26.2^{\mathrm{b}}$ & $40.5^{\mathrm{a}}$ \\
Interquartile range (\%) & $29.3-63.3$ & $21.0-39.0$ & $25.7-49.5$ \\
\hline
\end{tabular}

IVM: in vitro maturation.

${ }^{a b}$ Values with different superscripts are significantly different $(P<0.05)$.

Table 2. Comparison between normal unstimulated human ovaries (group 1a) and FSH-stimulated polycystic ovaries (group 2)

\begin{tabular}{lcc}
\hline & $\begin{array}{c}\text { Group 1a } \\
\text { (unstimulated) }\end{array}$ & $\begin{array}{c}\text { Group 2 } \\
\text { (stimulated) }\end{array}$ \\
\hline Number of cycles & 27 & 18 \\
Number of oocytes & 132 & 153 \\
Number of oocytes for IVM & 88 & 88 \\
Metaphase II (\%) & $51(58.0)$ & $59(67.1)$ \\
Two pronuclei (\% metaphase II) & $33(64.7)$ & $38(64.4)$ \\
Cleavage (\% metaphase II) & $19(37.3)$ & $30(50.8)$ \\
Embryos transferred & 17 & 25 \\
Implantation (\%) & $2(11.8)$ & $3(12.0)$ \\
Apoptotic index of granulosa cells (\%) & 45.9 & 40.5 \\
\hline
\end{tabular}

IVM: in vitro maturation.

There were no significant differences between groups.

A total of 132 oocytes was recovered from normal unstimulated ovaries and, of these, $88(67 \%)$ cumulusenclosed oocytes were used in the experiment. The maturation rate, fertilization rate, cleavage rate and implantation rate of oocytes from $\mathrm{FSH}$-primed polycystic ovaries were not significantly different from those of oocytes obtained from normal unstimulated ovaries (Table 2).

No correlation was found between maturation rate and apoptotic index for each ovary (data not shown).

The two ovaries of each patient undergoing regular menstruation in unstimulated cycles were grouped according to appearance of a dominant follicle on the day of aspiration. The incidence of apoptosis was significantly higher in granulosa cells from the ovary without a dominant follicle compared with granulosa cells from the ovary with a dominant follicle. The rates of maturation, fertilization and cleavage were not significantly different between the two groups (Table 3).

\section{Discussion}

Previous studies of human IVF showed that a low incidence of apoptosis in granulosa cells and cumulus cells resulted in a better outcome for the oocytes from the corresponding
Table 3. Comparison between human ovary with a dominant follicle and ovary without a dominant follicle on the day of aspiration (unstimulated normal ovaries)

\begin{tabular}{lcc}
\hline & $\begin{array}{c}\text { Ovary with a } \\
\text { dominant follicle }\end{array}$ & $\begin{array}{c}\text { Ovary without a } \\
\text { dominant follicle }\end{array}$ \\
\hline Number of ovaries & 27 & 27 \\
Number of oocytes & 62 & 70 \\
Oocytes for IVM (\%) & $36(51.1)$ & $52(74.3)$ \\
Metaphase II (\%) & $24(66.7)$ & $27(51.9)$ \\
2PN fertilization & $14(58.3)$ & $19(70.3)$ \\
Cleaved & $9(37.5)$ & $10(37.0)$ \\
Apoptotic index of & & $59.3^{\mathrm{b}}$ \\
\hline granulosa cells (\%) & $41.9^{\mathrm{a}}$ & \\
\hline
\end{tabular}

2PN fertilization: presence of two pronuclei.

ab Values with different superscripts are significantly different $(P<0.05$; analysed by Mann-Whitney $U$ test).

follicle (Nakahara et al., 1997b; Høst et al., 2000). However, there is little information about the importance and necessity of apoptosis in granulosa cells of immature human follicles. Høst et al. (2000) reported a significantly higher degree of apoptosis in cumulus cells from immature oocytes compared with cumulus cells from metaphase II oocytes when oocytes were obtained after controlled ovarian hyperstimulation. Maturation of these immature oocytes in vitro has demonstrated that their developmental competence is markedly lower than that of their counterparts matured in vivo (Kim et al., 2000). These oocytes may represent an inferior population as they failed to mature although the follicles were exposed to supraphysiological concentrations of gonadotrophins.

The aim of the present study was to obtain oocytes that have been able to develop into viable embryos after IVM in previous studies. There is evidence that competent oocytes in cows could originate from non-growing or early atretic follicles (Blondin et al., 1997). A reduced follicular growth has been shown to be advantageous before oocyte collection for IVM. In unstimulated cycles in humans the recovery of oocytes has to coincide with selection of the dominant follicle (Mikkelsen et al., 2000). In stimulated cycles in humans a time interval between FSH administration and aspiration improves the developmental capacity of oocytes (Mikkelsen et al., 1998). In the present study, the recovery of oocytes was performed according to these criteria.

In women undergoing regular menstruation, an increased percentage of granulosa cells from unstimulated follicles was apoptotic compared with granulosa cells from follicles stimulated with FSH before aspiration. It was not possible to examine the clinical significance with regard to apoptosis of granulosa cells and subsequent developmental competence in the present study, as the oocytes from FSH-primed normal ovaries and oocytes from unprimed normal ovaries were cultured in different culture media.

There are conflicting data regarding the maturation rate 
of in vitro-matured oocytes from normal ovaries retrieved after FSH-priming in vivo (Wynn et al., 1998; Mikkelsen et al., 1999). Wynn et al. (1998) reported an increased number of oocytes matured in vitro after FSH priming compared with women without priming. Mikkelsen et al. (1999) could not demonstrate any differences in the rates of maturation, fertilization or cleavage between the two groups.

Several studies report the use of in vitro-matured and fertilized oocytes in women with irregular cycles and PCOS (Trounson et al., 1998; Cha et al., 2000; Chian et al., 2000). The maturation rate of immature oocytes retrieved from patients with PCOS has been reported to be impaired compared with that of women undergoing regular cycles (Barnes et al., 1996). Suikkari et al. (2000) proposed priming with FSH before oocyte pick-up to compensate for this impaired maturation rate.

In the present study, the incidence of apoptosis of granulosa cells originating from $\mathrm{FSH}$-primed polycystic ovaries did not differ from the incidence of apoptosis of granulosa cells originating from unstimulated normal ovaries. Furthermore, the rates of maturation, fertilization and cleavage were not significantly different between the two groups. These findings support the concept that at least some antral follicles in the ovaries of women with PCOS are not committed irreversibly to atresia and, therefore, can be rescued by exogenous FSH.

There is conflicting evidence regarding the importance of a dominant follicle on the day of aspiration before IVM. Cobo et al. (1999) reported that once selection of the leading follicle has occurred, the developmental potential of the oocytes is impaired. The explanation for these findings should be a negative effect of the selection process. However, Whitacre et al. (1998) and Thorton et al. (1999) have raised some doubts about the significance of the dominant follicle. Selection of a dominant follicle from a growing cohort is controlled by feedback mechanisms between the ovary and the hypothalamic-pituitary axis and by intra-ovarian communication. Intra-ovarian communication can include an endocrine regulation as well as follicle-follicle interactions. Hence, a follicle can maintain dominance by inhibiting challengers (Macklon and Fauser, 2000). According to these findings, the oocytes from the ovary with a dominant follicle might be expected to be different from the oocytes originating from the ovary without a dominant follicle. It has been considered that the dominant follicle can be recognized by ultrasonography when it has reached $10 \mathrm{~mm}$ in diameter (Fauser and van Heusden, 1997). In the present study, we aimed to collect oocytes to coincide with selection of the dominant follicle. No differences in the rates of maturation, fertilization and cleavage were detected between oocytes originating from the ovary with a dominant follicle and oocytes originating from ovaries without a dominant follicle. No conclusion can be drawn with regard to implanted embryos because the embryos transferred originated from both ovaries in most of the patients.
A lower incidence of apoptosis was observed in the granulosa cells from the ovaries with a dominant follicle compared with ovaries without a dominant follicle. The explanation for this difference is unknown. For an individual patient undergoing a $\mathrm{GnRH}$ and human menopausal gonadotrophin (hMG) ovulation protocol, no difference in the incidence of apoptosis of one ovary over the other has been demonstrated (Moreira et al., 1999).

The data in the present study were measured on a per ovary basis and a higher incidence of granulosa cell apoptosis on a per ovary basis cannot be associated directly with each oocyte of compromised quality. Our results show an increased incidence of apoptosis in granulosa cells of immature follicles from unstimulated normal ovaries compared with granulosa cells from FSH-primed normal ovaries. In unstimulated cycles, it was found that follicles from the ovary with a dominant follicle had a decreased incidence of apoptosis compared with follicles from the ovary without a dominant follicle, whereas the rates of maturation, fertilization and cleavage were unaffected by a dominant follicle. Oocytes obtained from unstimulated normal ovaries did not differ from oocytes obtained from polycystic FSH-primed ovaries with respect to incidence of apoptosis of granulosa cells and maturation and fertility potential of the oocytes.

This study was supported in part by Medi-Cult.

\section{References}

Adams J, Frands S, Polson DW et al. (1985) Multifollicular ovaries: clinical and endocrine features and response to pulsative gonadotropinreleasing hormone Lancet 2 1375-1378

Barnes FL, Kausche A, Tiglias J, Wood C, Wilton L and Trounson A (1996) Production of embryos from in vitro-matured primary human oocytes Fertility and Sterility 65 1151-1156

Blondin P, Guilbault LA and Sirard MA (1997) The time interval between FSH-P administration and slaughter can influence the developmental competence of beef heifer oocytes Theriogenology 48 803-813

Cha KY, Han SY, Chung HM, Choi DH, Lim JM, Lee WS, Ko JJ and Yoon TK (2000) Pregnancies and deliveries after in vitro maturation culture followed by in vitro fertilization and embryo transfer without stimulation in women with polycystic ovary syndrome Fertility and Sterility 73 978-983

Chian RC, Buckett WM, Tulandi T and Tan SL (2000) Prospective randomized study of human chorionic gonadotrophin priming before immature oocyte retrieval from unstimulated women with polycystic ovarian syndrome Human Reproduction 15 165-170

Cobo AC, Requena A, Neuspiller F, Aragones M, Mercader A, Navarro J, Simón C, Remohí J and Pellicer A (1999) Maturation in vitro of human oocytes from unstimulated cycles: selection of the optimal day for ovum retrieval based on follicular size Human Reproduction 14 1864-1868

De Pol A, Vaccina F, Forabosco A, Cavazzutti E and Marzona L (1997) Apoptosis of germ cells during human prenatal oogenesis Human Reproduction 12 2235-2241

Driancourt M-A and Thuel B (1998) Control of oocyte growth and maturation by follicular cells and molecules present in follicular fluid: a review Reproduction, Nutrition, Development 38 345-362

Fauser BCJM and van Heusden AM (1997) Manipulation of human ovarian function: physiological concepts and clinical consequences Endocrine Reviews 18 71-106

Høst E, Lindenberg S, Kahn J and Christensen F (1999) DNA strand breaks in human sperm cells: a comparison between men with normal and 
oligozoospermic sperm samples Acta Obstetricia et Gynecologica Scandinavica 78 336-339

Høst E, Mikkelsen AL, Lindenberg S and Smidt-Jensen S (2000) Apoptosis in human cumulus cells in relation to maturation stage and cleavage of the corresponding oocyte Acta Obstetricia et Gynecologica Scandinavica 79 936-940

Kim B-K, Lee S-C, Kim K-J, Han C-H and Kim J-H (2000) In vitro maturation fertilization, and development of human germinal vesicle oocytes collected from stimulated cycles Fertility and Sterility 74 1153-1158

Macklon NS and Fauser BCJM (2000) Regulation of follicle development and novel approaches to ovaran stimulation for IVF Human Reproduction Update 6 307-312

Mikkelsen AL, Smith SD and Lindenberg S (1998) In-vitro maturation of immature human oocytes Human Reproduction 13 (Abstract Book 1 ) O-48

Mikkelsen AL, Smith SD and Lindenberg S (1999) In vitro maturation of human oocytes from regular menstruating women may be successfu without follicle stimulating hormone priming Human Reproduction $\mathbf{1 4}$ 1847-1851

Mikkelsen AL, Smith SD and Lindenberg S (2000) Impact of oestradiol and inhibin A concentrations on pregnancy rate in in-vitro oocyte maturation Human Reproduction 15 1685-1690

Moreira P, Saito H, Kaneko T, Saito T, Nakahara K and Hiroi M (1999) Are there any relationships between the fecundity of bilateral ovaries in an individual patient and the incidence of apoptotic granulosa cells? Human Reproduction 14 156-161

Nakahara K, Saito H, Saito T, Ito M, Ohta N, Sakai N, Tezuka N, Hiroi M and Watanabe $\mathbf{H}$ (1997a) Incidence of apoptotic bodies in membrana granulosa of the patients participating in an in vitro fertilization program Fertility and Sterility 67 302-308

Nakahara K, Saito H, Saito T, Ito M, Ohta N, Takahashi T and Hiroi M (1997b) The incidence of apoptotic bodies in membrana granulosa can predict prognosis of ova from patients participating in in vitro fertilization programs Fertility and Sterility $\mathbf{6 8} 312-317$

Oosterhuis GJE, Michgelsen HW, Lambalk CB, Schoemaker J and Vermes (1998) Apoptotic cell death in human granulosa-lutein cells: a possible indicator of in vitro fertilization outcome Fertility and Sterility $\mathbf{7 0}$ 747-749

Scott RT, Toner JP, Muasher SJ, Robinson S and Rosenwakzs Z (1989) Follicle stimulating hormone levels on cycle day 3 are predictive of in vitro fertilization outcome Fertility and Sterility $\mathbf{5 1}$ 651-654
Seifer DB, Gardiner AC, Ferreira KA and Peluso JJ (1996) Apoptosis as a function of ovarian reserve in women undergoing in-vitro fertilization Fertility and Sterility 66 593-598

Seifer DB, Lambert-Messerlian G, Hogan JW, Gardiner AC, Blazer AS and Berk CA (1997) Day 3 serum inhibin B is predictive of assisted reproductive technologies outcome Fertility and Sterility 67 110-114

Sirard MA, Picard L, Dery M, Coenen K and Blondin P (1999) The time interval between $\mathrm{FSH}$ administration and ovarian aspiration influences the development of cattle oocytes Theriogenology 51 699-708

Smith SD, Mikkelsen AL and Lindenberg S (2000) Development of human oocytes matured in vitro for 28 or 36 hours Fertility and Sterility 73 541-544

Suikkari A-M, Tulppala M, Tuuri T, Hovatta O and Barnes F (2000) Luteal phase start of low-dose FSH priming of follicles results in an efficient recovery, maturation and fertilization of immature human oocytes Human Reproduction 15 747-751

Thorton MH, Francis MM and Paulson RJ (1999) Immature oocyte retrieval: lessons from unstimulated IVF cycles Fertility and Sterility 70 647-650

Trounson A, Anderiesz C, Jones GM, Kausche A, Lolatgis N and Wood C (1998) Oocyte maturation Human Reproduction 13 (Supplement 3) $52-62$

Whitacre KS, Seifer DB, Friedman CI, Coskun S, Kennard EA, Kim MH and Alak BM (1998) Effects of ovarian source, patients age, and menstrua cycle phase on in vitro maturation of immature human oocytes Fertility and Sterility 70 1015-1021

Wu J, Zhang L and Wang X (2000) Maturation and apoptosis of human oocytes in vitro are age-related Fertility and Sterility 74 1137-1141

Wynn P, Picton HM, Krapez J, Rutherford AJ, Balen AH and Gosden RG (1998) Pretreatment with follicle stimulating hormone promotes the number of human oocytes reaching metaphase II by in vitro maturation Human Reproduction 13 3132-3138

Yuan W and Giudice LC (1997) Programmed cell death in human ovary is a function of follicle and corpus luteum status Journal of Clinical Endocrinology and Metabolism 31 3148-3155

Received 4 December 2000

First decision 22 January 2001.

Final revision received 19 March 2001.

Accepted 29 March 2001. 